\title{
UNIQUE-MAXIMUM AND CONFLICT-FREE COLORING FOR HYPERGRAPHS AND TREE GRAPHS*
}

\author{
PANAGIOTIS CHEILARIS ${ }^{\dagger}$, BALÁZS KESZEGH ${ }^{\ddagger}$, AND DÖMÖTÖR PÁLVÖLGYI ${ }^{\S}$
}

\begin{abstract}
We investigate the relationship between two kinds of vertex colorings of hypergraphs: unique-maximum colorings and conflict-free colorings. In a unique-maximum coloring, the colors are ordered, and in every hyperedge of the hypergraph the maximum color in the hyperedge occurs in only one vertex of the hyperedge. In a conflict-free coloring, in every hyperedge of the hypergraph there exists a color in the hyperedge that occurs in only one vertex of the hyperedge. We consider the corresponding unique-maximum and conflict-free chromatic numbers and investigate their relationship in arbitrary hypergraphs. Then, we concentrate on hypergraphs that are induced by simple paths in tree graphs.
\end{abstract}

1. Introduction. A hypergraph $H$ is a pair $(V, E)$, where $E$ (the hyperedge set) is a family of non-empty subsets of $V$ (the vertex set). A vertex coloring of a hypergraph $H=(V, E)$ is a function $C: V \rightarrow \mathbb{Z}^{+}$.

A hypergraph is a generalization of a graph. Therefore, it is natural to consider how to generalize proper vertex coloring of a graph to a vertex coloring of a hypergraph. (In a proper vertex coloring of a graph, any two vertices neighboring with an edge in the graph have to be assigned different colors by the coloring function $C$.) Vertex coloring in hypergraphs can be defined in many ways, so that when restricting the definition to simple graphs, it coincides with proper graph coloring.

At one extreme, it is only required that the vertices of each hyperedge are not all colored with the same color (except for singleton hyperedges). This is called a nonmonochromatic coloring of a hypergraph. The minimum number of colors necessary to color in such a way a hypergraph $H$ is the (non-monochromatic) chromatic number of $H$, denoted by $\chi(H)$.

At the other extreme, we can require that the vertices of each hyperedge are all colored with different colors. This is called a colorful or rainbow coloring of $H$ and we have the corresponding rainbow chromatic number of $H$, denoted by $\chi_{\mathrm{rb}}(H)$.

In this paper we investigate the following two types of vertex colorings of hypergraphs that are between the above two extremes.

Definition 1.1. A unique-maximum coloring of $H=(V, E)$ with $k$ colors is a function $C: V \rightarrow\{1, \ldots, k\}$ such that for each $e \in E$ the maximum color in $e$ occurs exactly once on the vertices of e. The minimum $k$ for which a hypergraph $H$ has a unique-maximum coloring with $k$ colors is called the unique-maximum chromatic number of $H$ and is denoted by $\chi_{\mathrm{um}}(H)$.

Definition 1.2. A conflict-free coloring of $H=(V, E)$ with $k$ colors is a function $C: V \rightarrow\{1, \ldots, k\}$ such that for each $e \in E$ there is a color in $e$ that occurs exactly once on the vertices of $e$. The minimum $k$ for which a hypergraph $H$ has a conflict-free coloring with $k$ colors is called the conflict-free chromatic number of $H$ and is denoted by $\chi_{\mathrm{cf}}(H)$.

We also introduce a new coloring, that proves useful in showing lower bounds, and that could be of independent interest.

*A preliminary version of this work appeared in the 38th International Conference on Current Trends in Theory and Practice of Computer Science (SOFSEM 2012).

${ }^{\dagger}$ Department of Informatics, Università della Svizzera italiana, Lugano, Switzerland.

¥Alfréd Rényi Institute of Mathematics, Budapest, Hungary.

$\S$ Eötvös University, Budapest, Hungary. 
Definition 1.3. An odd coloring of $H=(V, E)$ with $k$ colors is a function $C: V \rightarrow\{1, \ldots, k\}$ such that for each $e \in E$ there is a color that occurs an odd number of times on the vertices of $e$. The minimum $k$ for which a hypergraph $H$ has an odd coloring with $k$ colors is called the odd chromatic number of $H$ and is denoted by $\chi_{\text {odd }}(H)$.

We also introduce a useful tool to argue about odd colorings.

Definition 1.4 (Parity vector). Given a coloring $C: V \rightarrow\{1, \ldots, k\}$ and a set $e \subseteq V$, the parity vector of $e$ is an element of $\{0,1\}^{k}$ in which the $i^{\text {th }}$ coordinate equals the parity (0 or 1) of the number of elements in e colored with $i$.

REMARK 1.5. A coloring of a hypergraph is odd if and only if the parity vector of every hyperedge is not the all-zero vector.

Every rainbow coloring is unique-maximum, every unique-maximum coloring is conflict-free, and every conflict-free coloring is odd and non-monochromatic. Therefore, for every hypergraph $H, \max \left(\chi(H), \chi_{\text {odd }}(H)\right) \leq \chi_{\mathrm{cf}}(H) \leq \chi_{\mathrm{um}}(H) \leq \chi_{\mathrm{rb}}(H)$. Note that an odd coloring can be monochromatic.

The study of conflict-free coloring hypergraphs started in [9, 21], with an emphasis on hypergraphs induced by geometric shapes. The main application of conflict-free coloring is that it models a frequency assignment for cellular networks. A cellular network consists of two kinds of nodes: base stations and mobile agents. Base stations have fixed positions and provide the backbone of the network; they are represented by vertices in $V$. Mobile agents are the clients of the network and they are served by base stations. This is done as follows: Every base station has a fixed frequency; this is represented by the coloring $C$, i.e., colors represent frequencies. If an agent wants to establish a link with a base station it has to tune itself to this base station's frequency. Since agents are mobile, they can be in the range of many different base stations. To avoid interference, the system must assign frequencies to base stations in the following way: For any range, there must be a base station in the range with a frequency that is not used by some other base station in the range. One can solve the problem by assigning $n$ different frequencies to the $n$ base stations. However, using many frequencies is expensive, and therefore, a scheme that reuses frequencies, where possible, is preferable. Conflict-free coloring problems have been the subject of many recent papers due to their practical and theoretical interest (see e.g. [22, 19, 11, 7, 8, 1]).

Most approaches in the conflict-free coloring literature rely on the stronger uniquemaximum colorings (a notable exception is the 'triples' algorithm in [1]), because unique-maximum colorings are easier to argue about in proofs, due to their additional structure. Another advantage of unique-maximum colorings is the simplicity of computing the unique color in any range (it is always the maximum color), given a unique-maximum coloring, which can be helpful if very simple mobile devices are used by the agents.

Other hypergraphs that have been studied with respect to these colorings, are ones which are induced by a graph and (a) its neighborhoods or (b) its paths:

(a) Given a graph $G$, consider the hypergraph with the same vertex set as $G$ and a hyperedge for every distinct vertex neighborhood of $G$; such conflict-free colorings have been studied in $[5,18]$.

(b) Given a graph $G$, consider the hypergraph $H$ with the same vertex set as $G$ and a hyperedge for every distinct vertex set that can be spanned by a simple path of $G$. A unique-maximum (respectively conflict-free, odd) coloring of $H$ is called a unique-maximum (respectively conflict-free, odd) coloring of 
$G$ with respect to paths; we also define the corresponding graph chromatic numbers, $\chi_{\mathrm{um}}^{\mathrm{p}}(G)=\chi_{\mathrm{um}}(H), \chi_{\mathrm{cf}}^{\mathrm{p}}(G)=\chi_{\mathrm{cf}}(H)$ and $\chi_{\mathrm{odd}}^{\mathrm{p}}(G)=\chi_{\mathrm{odd}}(H)$. Sometimes to improve readability of the text, we simply talk about the UM (respectively CF, ODD) chromatic number of a graph.

Unique-maximum colorings with respect to paths of graphs are known alternatively in the literature as ordered colorings or vertex rankings, and the uniquemaximum chromatic number is also known as tree-depth [17]. The problem of computing such unique-maximum colorings is a well-known and widely studied problem (see e.g. [13]) with many applications including VLSI design [14] and parallel Cholesky factorization of matrices [15]. The problem is also interesting for the Operations Research community, because it has applications in planning efficient assembly of products in manufacturing systems [12]. In general, it seems that the vertex ranking problem can model situations where interrelated tasks have to be accomplished fast in parallel (assembly from parts, parallel query optimization in databases, etc.). For general graphs, finding the exact unique-maximum chromatic number with respect to paths of a graph is NP-complete $[20,16,2,17]$ and there is a polynomial time $O\left(\log ^{2} n\right)$ approximation algorithm [3], where $n$ is the number of vertices.

The paper [6] studied the relationship between the two graph chromatic numbers, $\chi_{\mathrm{um}}^{\mathrm{p}}(G)$ and $\chi_{\mathrm{cf}}^{\mathrm{p}}(G)$, showing that for every graph $G, \chi_{\mathrm{um}}^{\mathrm{p}}(G) \leq 2^{\chi_{\mathrm{cf}}^{\mathrm{p}}(G)}-1$, and providing a sequence of graphs for which the ratio $\chi_{\mathrm{um}}^{\mathrm{p}}(G) / \chi_{\mathrm{cf}}^{\mathrm{p}}(G)$ tends to 2 . Moreover, the authors of [6] proved that even checking whether a given coloring of a graph is conflict-free is coNP-complete (whereas the same problem for unique-maximum colorings is in $\mathrm{P}$ ).

Odd colorings with respect to paths of graphs have been recently studied in $[4,10]$, independently from our work. In these papers, they are called parity vertex colorings.

Our results. In this work, we study the relationship between unique-maximum and conflict-free colorings. Throughout this work, we use base 2 logarithms, which are denoted by "log".

First, we give an exact answer to the question "How much larger than $\chi_{\mathrm{cf}}(H)$ can $\chi_{\mathrm{um}}(H)$ be?" for a general hypergraph $H$. In section 2 , we show that if for a hypergraph $H, \chi_{\mathrm{cf}}(H)=k>1$, then $\chi_{\mathrm{um}}(H)$ is bounded from above, roughly, by $\frac{k-1}{k}|V|$, and this is tight; the result remains true even if we restrict ourselves to uniform hypergraphs.

In section 3 , we show that for every tree graph $T, \chi_{\mathrm{um}}^{\mathrm{p}}(T) \leq\left(\chi_{\mathrm{cf}}^{\mathrm{p}}(T)\right)^{3}$ and provide a sequence of trees for which the ratio $\chi_{\mathrm{um}}^{\mathrm{p}}(T) / \chi_{\mathrm{cf}}^{\mathrm{p}}(T)$ tends at least to $\log 3 \approx 1.58$ (corollary 3.29). In our proof, we employ a new parity vector argument, that could also be of independent interest (see definition 1.4 and theorem 3.6). We study trees because for general graphs the only known upper bound for $\chi_{\mathrm{um}}^{\mathrm{p}}(G)$ is exponential in $\chi_{\mathrm{cf}}^{\mathrm{p}}(G)$; see $[6]$.

Our results on trees have also implications for the relationship of the ODD and UM chromatic number of trees. In particular, corollary 3.29 disproves the following conjecture from [4]: "For any tree $T$ we have $\chi_{\mathrm{um}}^{\mathrm{p}}(T)-\chi_{\mathrm{odd}}^{\mathrm{p}}(T) \leq 1$ ". This conjecture was also disproved independently in [10], but our disproof is stronger in the following sense: in [10], the authors give a sequence of trees for which the ratio $\chi_{\mathrm{um}}^{\mathrm{p}}(T) / \chi_{\text {odd }}^{\mathrm{p}}(T)$ is at least 1.5, whereas our corollary 3.29 implies a sequence of trees for which the aforementioned ratio tends to at least 1.58. We also improve the trivial lower bound on the ODD chromatic number of binomial trees given in [10] (see remark 3.7).

Conclusions and open problems are presented in section 4 . 
2. General hypergraphs. In general, it is not possible to bound $\chi_{\mathrm{cf}}$ with a function of $\chi_{\text {odd }}$. For example, the hypergraph $H^{\prime}$ with hyperedge set consisting of all triples of $\{1, \ldots, n\}$ has $\chi_{\text {odd }}\left(H^{\prime}\right)=1$ and $\chi_{\mathrm{cf}}\left(H^{\prime}\right)=\lceil n / 2\rceil$. Although $\chi_{\mathrm{cf}}(H)=1$ implies $\chi_{\text {um }}(H)=1$, we can have a big gap as is shown by the following theorem.

TheOrEm 2.1. For a hypergraph $H$ on $n$ vertices, $\chi_{\mathrm{um}}(H) \leq n-\left\lceil n / \chi_{\mathrm{cf}}(H)\right\rceil+1$. Moreover, this is the best possible bound, i.e., for any positive integer $n$ there exists a hypergraph on $n$ vertices for which equality holds.

Proof. A simple algorithm achieving the upper bound is the following. Given a hypergraph $H$ with $\chi_{\mathrm{cf}}(H)=k$, take a conflict-free coloring of $H$ with $k$ colors, color the largest color class with color 1 , all the other vertices with all different colors (bigger than 1). It is not difficult to see that this is a unique-maximum coloring and that it uses at most $n-\lceil n / k\rceil+1$ colors.

For a given $n$ and $k$ equality holds for the hypergraph $H$ whose $n$ vertices are partitioned into $k$ (almost) equal parts, all of size $\lceil n / k\rceil$ or $\lfloor n / k\rfloor$, and its edges are all sets of size 2 and 3 covering vertices from exactly 2 parts. We have $\chi_{\mathrm{cf}}(H)=k$ because in any conflict-free coloring of $H$ there are no two vertices in different parts having the same color and $\chi_{\mathrm{um}}(H) \geq n-\lceil n / k\rceil+1$ because in any unique-maximum coloring of $H$ all vertices must have different colors except that the vertices of one part can be all colored with $1 . \square$

For uniform hypergraphs without small hyperedges, we can make the inequality tighter.

THEOREM 2.2. If $l \geq 3$ and $k \geq 2$ then for an arbitrary $l$-uniform hypergraph $H$ with $\chi_{\mathrm{cf}}(H)=k$ having $n \geq 2 k l$ vertices we have $\chi_{\mathrm{um}}(H) \leq n-\lceil n / k\rceil-l+4$. Moreover, this is the best possible bound, i.e., for arbitrary $n \geq 2 k l$ there exists a hypergraph for which equality holds.

Proof. The proof is similar to the proof of theorem 2.1, although more complicated.

For the first part of the theorem, we describe an algorithm that produces a uniquemaximum coloring with $n-\lceil n / k\rceil-l+4$ colors. Given an $l$-uniform hypergraph $H=(V, E)$ on $n$ vertices with $\chi_{\mathrm{cf}}(H)=k$, take a conflict-free coloring $C_{\mathrm{cf}}$ of $H$ using $k$ colors. Consider the $k$ color classes $X_{i}=C_{\text {cf }}^{-1}(i)$, for $i \in\{1, \ldots, k\}$, and without loss of generality assume they are in order of non-increasing size, i.e.,

$$
\left|X_{1}\right| \geq\left|X_{2}\right| \geq \cdots \geq\left|X_{k}\right|
$$

Now, consider the following coloring $C_{\mathrm{um}}$ : Color the vertices of $X_{1}$ with color 1 , color $\min \left(l-2,\left|X_{2}\right|\right)$ vertices of $X_{2}$ with color 2 , and color all other vertices with all different colors. Observe, first of all, that $C_{\text {um }}$ is a conflict-free coloring, because it is a refinement of conflict-free coloring $C_{\mathrm{cf}}$. We additionally prove that $C_{\mathrm{um}}$ is a unique-maximum coloring. Indeed, for an arbitrary edge $e$, if the maximum color occurring in $e$ is greater than 2, then $e$ has the unique-maximum property, because each color greater than 2 occurs in exactly one vertex of the hypergraph. Otherwise, the only colors that occur in $e$ are 1 and 2 . Since $|e|=l$ and color 2 occurs in at most $l-2$ vertices of $e$, color 1 occurs in at least two vertices of $e$. But then, $e$ has the conflict-free property if and only if exactly one vertex of $e$ is colored with 2, i.e., $e$ has the unique-maximum property.

The number of colors used in $C_{\mathrm{um}}$ is $2+n-\left|X_{1}\right|-\min \left(l-2,\left|X_{2}\right|\right)$. If $\left|X_{2}\right| \geq l-2$ then (also because $\left|X_{1}\right| \geq\lceil n / k\rceil$ ) this number is at most $n-\lceil n / k\rceil-l+4$. Otherwise, $\left|X_{2}\right|<l-2$ and in that case the number of colors used is $2+n-\left|X_{1}\right|-\left|X_{2}\right|$. Using 
inequalities $\left|X_{1}\right| \geq\lceil n / k\rceil$ and $\left|X_{1}\right|+(k-1)\left|X_{2}\right| \geq n$, we get

$$
\left|X_{1}\right|+\left|X_{2}\right| \geq \frac{1}{k-1}\left(n+(k-2)\left\lceil\frac{n}{k}\right\rceil\right)=\left\lceil\frac{n}{k}\right\rceil+\frac{1}{k-1}\left(n-\left\lceil\frac{n}{k}\right\rceil\right) .
$$

Then, using inequality $\left\lceil\frac{n}{k}\right\rceil<\frac{n}{k}+1$, we get

$$
\left|X_{1}\right|+\left|X_{2}\right|>\left\lceil\frac{n}{k}\right\rceil+\frac{1}{k-1}\left(n-\frac{n}{k}-1\right)=\left\lceil\frac{n}{k}\right\rceil+\frac{n}{k}-\frac{1}{k-1} \geq\left\lceil\frac{n}{k}\right\rceil+\frac{n}{k}-1 .
$$

Finally, using inequality $n \geq 2 k l$, we get

$$
\left|X_{1}\right|+\left|X_{2}\right| \geq\left\lceil\frac{n}{k}\right\rceil+2 l-1 \geq\left\lceil\frac{n}{k}\right\rceil+l-2 .
$$

Thus, the number of colors used is $2+n-\left|X_{1}\right|-\left|X_{2}\right| \leq n-\lceil n / k\rceil-l+4$.

For the second part of the theorem, given $k, l$, and $n$ with $n \geq 2 k l$, we construct an $l$-uniform hypergraph $H$ with $\chi_{\mathrm{cf}}(H)=k$ and $\chi_{\mathrm{um}}(H)=n-\lceil n / k\rceil-l+4$. We have $n$ vertices partitioned into $k$ almost equal parts $V_{1}, V_{2}, \ldots, V_{k}$, the first $k^{\prime}$ having size $\lceil n / k\rceil$, the rest having size $\lceil n / k\rceil-1$. The hyperedge set of $H$ consists of all hyperedges of size $l$ for which there is a part $V_{i}$ that intersects the edge in exactly one vertex. During the rest of the proof we will use several times the pigeonhole principle on the above defined parts.

It is not difficult to see that the coloring defined by the partition $V_{1}, \ldots, V_{k}$ is a conflict-free coloring using $k$ colors, i.e., $\chi_{\mathrm{cf}}(H) \leq k$. We now prove that there is no conflict-free coloring using less than $k$ colors. For that, take a conflict-free coloring $C_{\text {cf }}$ of $H$ with the optimal number of colors.

For a color $c$, if its color class $C_{\mathrm{cf}}^{-1}(c)$ is covered by some $V_{i}$ then its size is at most $\left|V_{i}\right|$. Thus, we have at most $k^{\prime}$ such color classes of size $\lceil n / k\rceil$ and the rest is of size at most $\lceil n / k\rceil-1$. For a color $c$ for which its color class $C_{\mathrm{cf}}^{-1}(c)$ is not covered by one part of the partition, $C_{\mathrm{cf}}^{-1}(c)$ must intersect at least two different parts, $V_{i}$ and $V_{j}$ with $i \neq j$. If $\left|C^{-1}(c)\right|>2 l-4$ then either there is an $l$-subset of $C^{-1}(c)$ having exactly one point from $V_{i}$ or there is an $l$-subset of $C^{-1}(c)$ having exactly one point from $V_{j}$, which is a contradiction as these $l$-subsets would be monochromatic edges of $H$. If $\left|C_{\mathrm{cf}}^{-1}(c)\right| \leq 2 l-4$, then our assumption $n \geq 2 k l$ implies $2 l-4<\lceil n / k\rceil-1$, i.e., if a color class is not covered by some $V_{i}$ then it is smaller than $\lceil n / k\rceil-1$. Hence, the only way we can color all the vertices using only $k$ colors is by not having color classes intersecting two parts and by having every color class equal to a part of the partition. Thus, we proved that if $n \geq 2 k l$ then $\chi_{\mathrm{cf}}(H)=k$ and also that the only optimal coloring is the one defined by the partition.

Now, take a unique-maximum coloring $C_{\mathrm{um}}$ with the optimal number of colors. We prove that it uses at least $n-\lceil n / k\rceil-l+4$ colors. We define $c$ to be the biggest color for which there are at least 2 vertices having color $c$. By definition every color bigger than $c$ is used only at most once in this unique-maximum coloring. We define $Y$ to be the set of vertices with color $c$ and $Y^{\prime}$ to be the set of vertices with color $c$ or smaller.

Observation 2.3. Coloring $C_{\text {um }}$ uses $n-\left|Y^{\prime}\right|+c$ colors.

Since every edge has the unique-maximum property, the following is true.

OBSERVATION 2.4. There is no edge that contains only vertices from $Y^{\prime}$ and contains at least two vertices from $Y$.

If $Y^{\prime}$ can be covered by some $V_{i}$, then $\left|Y^{\prime}\right| \leq\lceil n / k\rceil$ and so we used at least $n-\lceil n / k\rceil+1 \geq n-\lceil n / k\rceil-l+4$ colors altogether. If $Y^{\prime}$ cannot be covered by one $V_{i}$ of the partition, then we have 3 cases: 
(i) $Y$ cannot be covered by one part.

In this case there are two vertices with color $c$ that are in different parts, $x$ in $V_{i}$ and $y$ in $V_{j}$ with $i \neq j$. If $\left|Y^{\prime}\right|>2 l-4$, then there is an $l$-subset of $Y^{\prime}$ containing only $x$ from $V_{i}$ and $l-1$ vertices from other parts (including $y$ ) or an $l$-subset of $Y^{\prime}$ containing only $y$ from $V_{j}$ and $l-1$ vertices from other parts (including $x$ ). Any of these two subsets would be an edge of $H$ contradicting observation 2.4. Thus, $\left|Y^{\prime}\right| \leq 2 l-4$ and so we used at least $n-(2 l-4)+1 \geq n-\lceil n / k\rceil-l+4$ colors (for the last inequality we used that $n \geq 2 k l)$.

In the next two cases, as $Y$ is contained in some $V_{i}$, but $Y^{\prime}$ is not, we have $Y^{\prime} \neq Y$ and thus $c \geq 2$.

(ii) $Y$ is contained in some $V_{i}$ and $Y^{\prime}$ can be covered by two parts $V_{i}$ and $V_{j}$. If $\left|Y^{\prime} \cap V_{i}\right|>l-2$ then there exists an $l$-subset of $Y^{\prime}$ containing exactly one vertex from $V_{j}$ and $l-1$ vertices from $V_{i}$ such that at least two of these vertices have color $c$. This subset would be an edge of $H$ contradicting observation 2.4. Thus, $\left|Y^{\prime}\right| \leq\left|V_{j}\right|+l-2 \leq\lceil n / k\rceil+l-2$, and as $c \geq 2$, we used at least $n-(\lceil n / k\rceil+l-2)+2=n-\lceil n / k\rceil-l+4$ colors.

(iii) $Y$ is contained in some $V_{i}$ and $Y^{\prime}$ cannot be covered by two parts.

In this case $Y^{\prime}$ contains points from at least three parts, $V_{i}, V_{j}$ and some $V_{h}$. Now, it is easy to see that if $\left|Y^{\prime}\right|>2 l-6$ then there exists an $l$-subset of $Y^{\prime}$ containing at least two vertices from $V_{i}$ with color $c$ and that either has exactly one vertex from $V_{j}$ or exactly one vertex from $V_{h}$. This subset would be an edge in $H$ contradicting observation 2.4. Thus, $\left|Y^{\prime}\right| \leq 2 l-6$, and as $c \geq 2$, we used at least $n-(2 l-6)+2 \geq n-\lceil n / k\rceil-l+4$ colors.

3. Tree graphs. In this section, to ease readability, we use UM for $\chi_{\mathrm{um}}^{\mathrm{p}}, \mathrm{CF}$ for $\chi_{\mathrm{cf}}^{\mathrm{p}}$ and ODD for $\chi_{\mathrm{odd}}^{\mathrm{p}}$. We first give a simple observation.

OBSERVATION 3.1. Each of the graph chromatic numbers UM, CF, and ODD, is monotone with respect to subgraphs, i.e., if $H \subseteq G$, then $\operatorname{UM}(H) \leq \operatorname{UM}(G)$, $\mathrm{CF}(H) \leq \mathrm{CF}(G)$, and $\mathrm{ODD}(H) \leq \mathrm{ODD}(G)$.

Proof. A subgraph $H$ of a graph $G$ contains a subset of the paths of $G$. $\square$

We denote by $P_{n}$ the path graph with $n$ vertices. As a warm-up, we prove a simple claim about the odd chromatic number of the path graph. Our proof is a showcase of a parity vector argument, which we are going to also use later. For completeness, we include a computation of the conflict-free and unique-maximum chromatic numbers of the path graph [9].

Claim 3.2. For $n \geq 1, \operatorname{ODD}\left(P_{n}\right)=\mathrm{CF}\left(P_{n}\right)=\mathrm{UM}\left(P_{n}\right)=\lceil\log (n+1)\rceil$.

Proof. It is easy to see that $\operatorname{UM}\left(P_{n}\right) \leq\lceil\log (n+1)\rceil$ : assign the biggest color only to a median vertex of the path and then use recursion. Since we know that $\mathrm{UM}\left(P_{n}\right) \geq \mathrm{CF}\left(P_{n}\right) \geq \operatorname{ODD}\left(P_{n}\right)$, it is enough to prove that $2^{\mathrm{ODD}\left(P_{n}\right)}>n$. Take the $n$ paths starting from one endpoint. If there were two with the same parity vector, their symmetric difference (which is also a path) would contain an even number of each color. Thus, we have at least $n$ different parity vectors, none of which is the all-zero vector. But the number of non-zero parity vectors is at most $2^{\mathrm{ODD}\left(P_{n}\right)}-1$.

3.1. Upper bound for unique-maximum number of binary trees. We denote by $B_{d}$ the (rooted) complete binary tree with $d$ levels (and $2^{d}-1$ vertices). By convention, $B_{0}$ is the empty graph. It is easy to see that $\operatorname{UM}\left(B_{d}\right)=d$; for an optimal unique-maximum coloring, color the leaves of $B_{d}$ with color 1 , their parents with color 2, and so on, until you color the root with color $d$; for a matching lower 
bound, use induction on $d$. In this section, we prove an upper bound for $\operatorname{UM}\left(B_{d}\right)$ that is quadratic on $\mathrm{CF}\left(B_{d}\right)$. In fact, we will prove a stronger statement, that is, a bound for $\operatorname{UM}\left(B_{d}\right)$ that is quadratic on $\operatorname{ODD}\left(B_{d}\right)$. Moreover, instead of proving a bound just for complete binary trees, we are going to prove a bound for subdivisions of complete binary trees, because we will need that later in subsection 3.2. We first need the following definitions.

Definition 3.3. A graph $H$ is a subdivision of $G$ if $H$ is obtained by substituting some edges uv of $G$ by a path of new internal vertices between $u$ and $v$. The original vertices of $G$ in $H$ are called branch vertices.

Definition 3.4. Given is a rooted tree $T$ and a rooted subtree $T^{\prime}$ of $T$. We say that $T^{\prime}$ is compatible with $T$ if the closest vertex of $T^{\prime}$ to the root of $T$ is the root of $T^{\prime}$. (This is equivalent to the following: Any two vertices of $T^{\prime}$ have the same ancestor-descendant relation in both $T^{\prime}$ and $T$.)

We are now ready to state the following useful lemma.

Lemma 3.5. Let $B^{*}$ be a subdivision of $B_{d}$. Suppose we color (without any restrictions) the vertices of $B^{*}$ with $k$ colors. Then, there exists a vector $a=\left(a_{1}, a_{2}, \ldots, a_{k}\right)$ such that $\sum_{i=1}^{k} a_{i} \geq d$ and for every $i \in\{1, \ldots, k\}, B^{*}$ contains a subdivision $T^{i}$ of $B_{a_{i}}$ such that (1) $T^{i}$ is compatible with $B^{*}$ and (2) the branch vertices of $T^{i}$ are all colored with $i$.

Proof. We construct the vector $a$ and the subdivisions $\left\{T^{i}\right\}_{i=1}^{k}$ by induction on $d$.

For $d=1, B^{*}$ has exactly one vertex $v$, say with color $j$. Then, $a$ is such that $a_{j}=1$ and every other coordinate is $0 . T^{j}$ is the single (and branch) vertex $v$ (colored with $j$ and trivially compatible with $B^{*}$ ) and $T^{i}$ for $i \neq j$ is empty. We also have $\sum_{i=1}^{k} a_{i}=1 \geq 1$.

For $d>1$, consider the tree $B^{*}$ rooted at the branch vertex $v$ that corresponds to the root of $B_{d}$. Each of the left and right subtrees of $v$ contains a subdivision of $B_{d-1}$, which is compatible with $B^{*}$. Call these subdivisions $B^{\prime}$ and $B^{\prime \prime}$, for the left and right subtree, respectively. Thus, by the inductive hypothesis, we have vector $a^{\prime}$ and subdivisions $\left\{T^{\prime i}\right\}_{i=1}^{k}$ with $\sum_{i=1}^{k} a_{i}^{\prime} \geq d-1$ for $B^{\prime}$ and vector $a^{\prime \prime}$ and subdivisions $\left\{T^{\prime \prime i}\right\}_{i=1}^{k}$ with $\sum_{i=1}^{k} a_{i}^{\prime \prime} \geq d-1$ for $B^{\prime \prime}$.

- If $a^{\prime} \neq a^{\prime \prime}$, then define $a$ such that $a_{i}=\max \left(a_{i}^{\prime}, a_{i}^{\prime \prime}\right)$ for $i \in\{1, \ldots, k\}$, and define $T^{i}$ to be $T^{\prime i}$ or $T^{\prime \prime i}$ depending on whether $a_{i}^{\prime}$ or $a_{i}^{\prime \prime}$ is bigger.

- If $a^{\prime}=a^{\prime \prime}$, then define $a$ such that $a_{j}=a_{j}^{\prime}+1$ for the color $j$ of the root $v$ and $a_{i}=a_{i}^{\prime}$ for $i \neq j$. Also, define $T^{j}$ to be the subdivision of $B_{a_{j}}$ with root and new branch vertex $v$ that we obtain by connecting the roots of $T^{\prime j}$ and $T^{\prime \prime j}$ with a path in $B^{*}$. Branch vertices of $T^{\prime j}$ and $T^{\prime \prime j}$ are also branch vertices of $T^{j}$. By induction, all branch vertices of $T^{j}$ are colored with $j$ and $T^{j}$ is compatible with $B^{*}$. For $i \neq j$, define $T^{i}$ as $T^{\prime i}$.

In both cases, $\sum_{i=1}^{k} a_{i} \geq \min \left(\sum_{i=1}^{k} a_{i}^{\prime}, \sum_{i=1}^{k} a_{i}^{\prime \prime}\right)+1 \geq(d-1)+1=d$.

Theorem 3.6. For $d \geq 1$ and for every subdivision $B^{*}$ of $B_{d}, \operatorname{ODD}\left(B^{*}\right) \geq \sqrt{d}$.

Proof. Fix an optimal odd coloring with $k$ colors and consider the vector $a=$ $\left(a_{1}, \ldots, a_{k}\right)$ from lemma 3.5. Since $\sum_{i=1}^{k} a_{i} \geq d$, by the pigeonhole principle, there exists a color $i \in\{1, \ldots, k\}$ for which we have $a_{i} \geq d / k$.

Consider the $2^{a_{i}-1}$ paths that originate in a leaf of the $B_{a_{i}}$ subdivision and end in its root branch vertex. We claim that the parity vectors of the $2^{a_{i}-1}$ paths must be all different. Indeed, if there were two paths with the same parity vector, then the symmetric difference of the paths plus their lowest common vertex would form a path where the parity of each color is even, except maybe for color $i$, but since this new 
path starts and ends with color $i$, deleting any of its ends yields a path whose parity vector is the all-zero vector, a contradiction.

There are at most $2^{k}-1$ parity vectors, thus $2^{k}-1 \geq 2^{a_{i}-1} \geq 2^{\lceil d / k\rceil-1}$. From this we get $k>\lceil d / k\rceil-1$ which is equivalent to $k \geq\lceil d / k\rceil$ using the integrality. Thus, $k \geq \sqrt{d}$.

REMARK 3.7. The (rooted) binomial tree $T_{d}$ with $2^{d-1}$ vertices is defined as follows: $T_{1}$ is a single vertex; for $d>1, T_{d}$ consists of two disjoint copies of $T_{d-1}$ and an edge between their two roots, whereas the root of $T_{d}$ is the root of the first copy. These trees are used in [4, 10] and play a similar role to binary trees in our work. It is not difficult to prove by induction that $T_{2 d-1} \supseteq B_{d}$. As a result, theorem 3.6 implies $\operatorname{ODD}\left(T_{d}\right)=\Omega(\sqrt{d})$, which improves the trivial lower bound $\operatorname{ODD}\left(T_{d}\right)=\Omega(\log d)$ from [10].

3.2. Upper bound for unique-maximum chromatic number of arbitrary trees. We will try to find either a long path or a subdivision of a deep complete binary tree in every tree with high UM chromatic number. For this, we need the notion of UM-critical trees and their characterization from [13].

Definition 3.8. A graph is UM-critical, if the UM chromatic number of any of its subgraphs is smaller than its UM chromatic number. We also say that a graph is $k$-UM-critical, if it is UM-critical and its UM chromatic number equals $k$.

EXAMPLE 3.9. The complete graph $K_{k}$ and the path with $2^{k-1}$ vertices are both $k$-UM-critical. For $k \leq 3$ there is a unique $k$-UM-critical tree, the path with $2^{k-1}$ vertices. Consider the following tree $T$ on 8 vertices: Take two copies of $P_{4}$ and draw an edge from an internal vertex of one $P_{4}$ to an internal vertex of the other $P_{4}$. Tree $T$ is 4-UM-critical and $\mathrm{CF}(T)=3$ (see figure 3.1). ( $T$ is the smallest tree where the $C F$ and $U M$ chromatic numbers differ.)
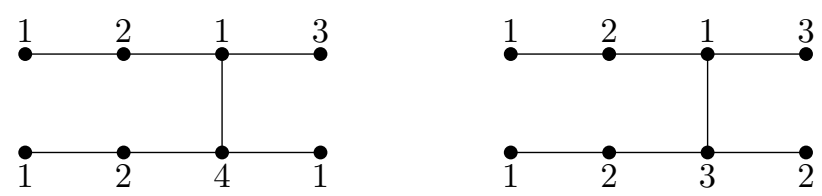

FIG. 3.1. Tree $T$ with optimal UM and CF colorings

TheOREm 3.10 (Theorem 2.1 in [13]). For $k>1$, a tree is $k$-UM-critical if and only if it has an edge that connects two $(k-1)$-UM-critical trees.

REMARK 3.11. A $k$-UM-critical tree has exactly $2^{k-1}$ vertices and the connecting edge must always be the central edge of the tree, i.e., the edge whose removal disconnects the tree into two subtrees with the same number of vertices. This implies that there is a unique way to partition the vertices of the $k$-UM-critical tree to two sets of vertices, each inducing a $(k-1)$-UM-critical tree, and so on.

Now we can define the structure trees of UM-critical trees, this is a new notion introduced by us.

Definition 3.12. For $l \in\{0, \ldots, k-1\}$, the $l$-deep structure tree of a $k$-UMcritical tree is the tree graph with a vertex for every one of the $2^{l}(k-l)$-UM-critical subtrees that we obtain by repeatedly applying theorem 3.10, and an edge between two vertices if the corresponding $(k-l)$-UM-critical subtrees have an edge between them in the $k$-UM-critical tree.

EXAMPLE 3.13. The 0-deep structure tree of any UM-critical tree is a vertex. The 1-deep structure tree of any UM-critical tree is an edge. The 2-deep structure 
tree of any UM-critical tree is a path with 4 vertices. The $(k-1)$-deep structure tree of a $k$-UM-critical tree is itself.

REMARK 3.14. It is not difficult to prove that the l-deep structure tree of a $U M$-critical tree is an $(l+1)$-UM-critical tree.

We start with a few simple observations that will be useful later to prove stronger statements.

ObSERVATION 3.15. If an $(l+1)$-UM-critical tree has no vertex of degree at least 3 , then it is the path with $2^{l}$ vertices.

Proof. Delete the central edge and use induction.

OBSERVATION 3.16. If an $(l+2)$-UM-critical tree has only one vertex of degree at least 3 , then it contains a path with $2^{l}$ vertices that ends in this vertex.

Proof. After deleting its central edge, one of the resulting $(l+1)$-UM-critical trees must be a path that was connected to the rest of the graph with one of its ends, thus we can extend it until the high degree vertex. $\mathrm{\square}$

OBSERVATION 3.17. If a tree contains two non-adjacent vertices with degree at least 3 , then it contains a subdivision of $B_{3}$.

Proof. The non-adjacent degree 3 vertices will be the second level of the binary tree, and any vertex on the path connecting them the root.

Claim 3.18. An $(l+2)$-UM-critical tree contains a path with $2^{l}$ vertices or a subdivision of $B_{3}$.

Proof. Because of the previous observations, we can suppose that our tree has exactly two vertices with degree at least 3 and these are adjacent. If the central edge is not the one between these vertices, then the graph must contain an $(l+1)$-UMcritical subgraph without any vertex with degree at least 3 , thus it is the path with $2^{l}$ vertices because of observation 3.15. If it connects the two high degree vertices, then, using observation 3.16 , we have two paths with $2^{l-1}$ vertices in the $(l+1)$-UM-critical subgraphs obtained by deleting the central edge ending in these vertices, thus with the central edge they form a path with $2^{l}$ vertices.

We are now ready to prove our main lemma, before the proof of the upper bound.

Lemma 3.19. For $k \geq 3$ and any $l$, every $k$-UM-critical tree contains a path with $2^{l}$ vertices or a subdivision of $B_{\left\lceil\frac{k+l+3}{l+2}\right\rceil}$.

Proof. The proof is by induction on $k$. For $3 \leq k \leq l+1$, the statement is true since $B_{2}=P_{3}$. For $l+2 \leq k \leq 2 l+3$, the statement is equivalent to our claim 3.18. For $k>2 l+3$, take the $(l+2)$-deep structure tree $S$ of the tree. If $S$ does not contain a path with $2^{l}$ vertices, then, using claim 3.18, $S$ contains a subdivision of $B_{3}$. Every one of the four leaf branch vertices of the above $B_{3}$ subdivision corresponds to a $(k-l-2)$-UM-critical subtree of the original tree. By induction, each one of the above four subtrees must contain a path with $2^{l}$ vertices or a subdivision of the complete binary tree with $\left\lceil\frac{k-l-2+l+3}{l+2}\right\rceil=\left\lceil\frac{k+l+3}{l+2}\right\rceil-1$ levels. If any of them contains the path, we are done. If each one of them contains a $B_{\left\lceil\frac{k+l+3}{l+2}\right\rceil-1}$ subdivision, then for every one of the four leaves, we can connect at least one of the two disjoint $B_{\left\lceil\frac{k+l+3}{l+2}\right\rceil-2}$ subdivisions of the $B_{\left\lceil\frac{k+l+3}{l+2}\right\rceil-1}$ subdivision in the leaf (as in figure 3.2, where each of the four relevant $B_{\frac{k+l+3}{l+2}-2}$ subdivisions and the paths connecting them are shown with heavier lines) to obtain a subdivision of a complete binary tree with $\left\lceil\frac{k+l+3}{l+2}\right\rceil-2+2$ levels, thus we are done.

Theorem 3.20. For every tree $T, \operatorname{ODD}(T) \geq(\operatorname{UM}(T))^{\frac{1}{3}}-O(1)$.

Proof. If $\operatorname{UM}(T)=k$, then $T$ contains a $k$-UM-critical tree, which (according to 


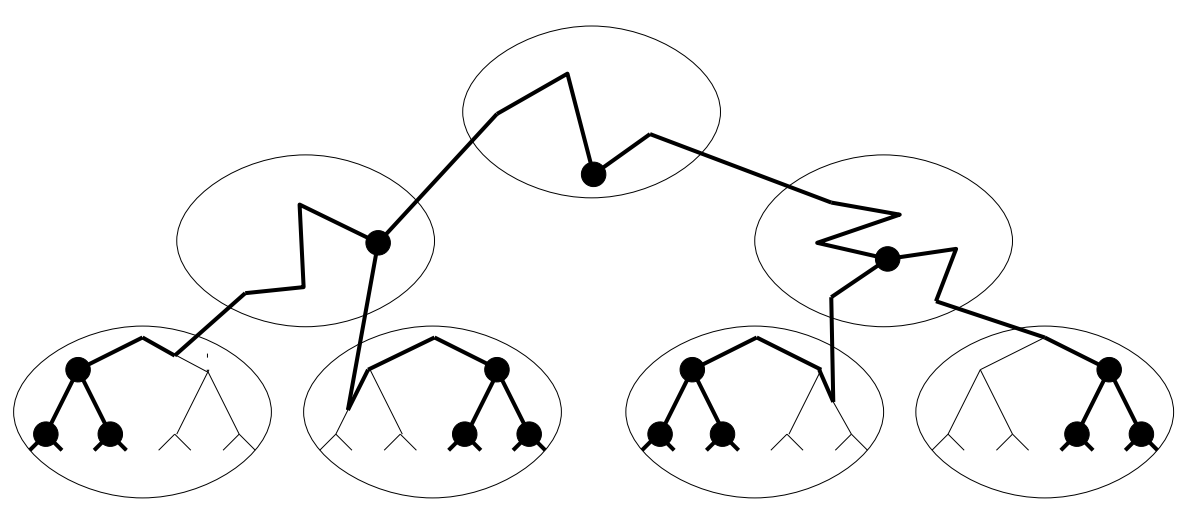

FIG. 3.2. Constructing a deep binary tree using induction for structure trees

lemma 3.19) contains a $P_{2^{l}}$ or a subdivision $B^{*}$ of $B_{\left\lceil\frac{k+l+3}{l+2}\right\rceil}$. Using monotonicity of ODD with respect to subgraphs (observation 3.1), together with $\operatorname{ODD}\left(P_{2^{l}}\right)=l+1$ (claim 3.2) and $\operatorname{ODD}\left(B^{*}\right) \geq \sqrt{\frac{k+l+3}{l+2}}$ (from theorem 3.6), we get $\operatorname{ODD}(T) \geq \max (l+$ $\left.1, \sqrt{\frac{k+l+3}{l+2}}\right)$. Choosing $l$ to be the closest integer to the solution of $l+1=\sqrt{\frac{k+l+3}{l+2}}$, we get $l=k^{\frac{1}{3}}+\Theta(1)$. Therefore, $\operatorname{ODD}(T) \geq(\operatorname{UM}(T))^{\frac{1}{3}}-O(1)$.

3.3. Trees with different unique-maximum and conflict-free numbers. We have seen that $\operatorname{UM}\left(B_{d}\right)=d$. We intend to show conflict-free colorings of some complete binary trees that use substantially less colors. We start with a simple example demonstrating our method.

Claim 3.21. $\mathrm{CF}\left(B_{7}\right) \leq 6$.

Proof. See figure 3.3. Color the root with 1, the second level with 2. Deleting the colored vertices leaves four $B_{5}$ subtrees. In each of these subtrees, every level will be monochromatic. From top to bottom, in the first use the colors $3,4,5,1,2$, in the second $4,5,6,1,2$, in the third $5,6,3,1,2$ and in the forth $6,3,4,1,2$. It is not difficult to verify that this is indeed a conflict-free coloring (but it will also follow from later results). Observe that in the top 2 levels 2 colors are used, in the next 3 levels 4 colors, and in the last 2 levels the same 2 colors are used as the ones in the top level.

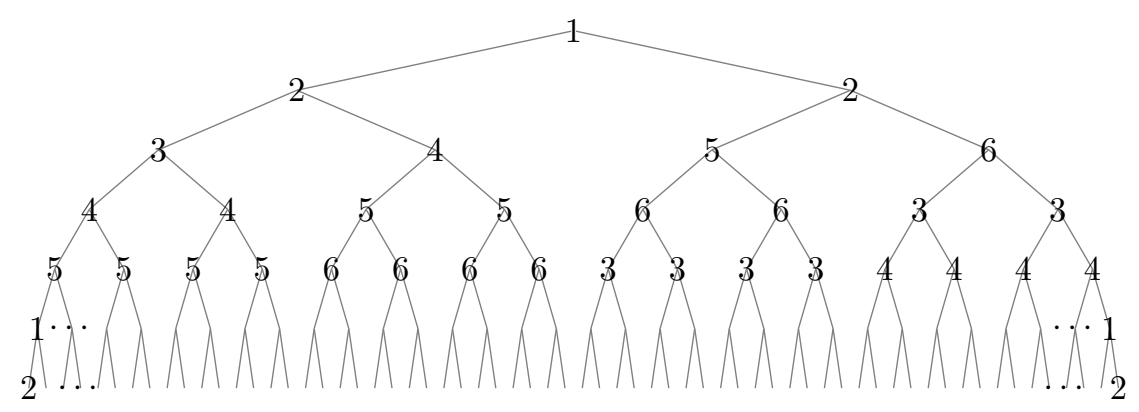

FIG. 3.3. An optimal $C F$ coloring of $B_{7}$

Corollary 3.22. $\mathrm{CF}\left(B_{2(r+1)+3 r}\right) \leq 4 r+2$. 
Proof. In the previous construction, every leaf had color 2 and their parents had color 1. Every such three vertex part can be the top of a new tree, similar to the original, and replacing 3, 4,5,6 with four new colors. This gives a tree with 12 levels and 10 colors. It is not difficult to verify that this is indeed a conflict-free coloring (but it will also follow from later results). Repeatedly applying this procedure, so that we have colors 1, 2 appearing in $2(r+1)$ levels and $r$ disjoint sets of 4 colors each, we get a coloring of $B_{2(r+1)+3 r}$ using $4 r+2$ colors.

To examine more closely why these colorings are conflict-free, we need to define some notions.

Definition 3.23. An ordered set is a sequence in which no element repeats. Two ordered sets are equal as sets if they have the same elements (ignoring the order of elements).

Definition 3.24. A family $\mathcal{F}$ of ordered sets is said to be prefix set-free, if any prefix of any ordered set in $\mathcal{F}$ is different from any other ordered set in $\mathcal{F}$ as a set (without the ordering). If the ground set has $n$ elements, every sequence has length at least $k$, and the cardinality of $\mathcal{F}$ is at least $2^{d}$, then we say that $\mathcal{F}$ is $a[k, d, n] \mathrm{PSF}$ family.

Example 3.25. $\{\langle 1,3\rangle,\langle 1,2,3\rangle\}$ is a $[2,1,3]$ PSF family and $\{\langle 1\rangle,\langle 2,1\rangle,\langle 2,3\rangle$, $\langle 3,1\rangle,\langle 3,1,2\rangle\}$ is a $[1,2,3]$ PSF family but $\{\langle 2,1\rangle,\langle 1,2,3\rangle\}$ is not a PSF family.

Claim 3.26. For any $[k, d, n]$ PSF family $d \leq \log \sum_{i=k}^{n}\left(\begin{array}{l}n \\ i\end{array}\right)$.

Proof. Any two ordered sets in the PSF family must differ as sets. $\square$

Claim 3.27. There is a $[k, d, n]$ PSF family with $d=\left\lfloor\log \left(\begin{array}{l}n \\ k\end{array}\right)\right\rfloor$.

Proof. Take all $k$-element subsets of $\{1, \ldots, n\}$ and order each arbitrarily.

Since these bounds do not differ much if $k>\left(\frac{1}{2}+\epsilon\right) n$, we do not attempt to get sharper bounds.

THEOREM 3.28. If there is a $[k, d, n]$ PSF family where the size of every set is at most $k+d$, then $\mathrm{CF}\left(B_{d(r+1)+k r}\right) \leq n r+d$.

Proof. First, we show that $\mathrm{CF}\left(B_{k+2 d}\right) \leq n+d$. Color the top $d$ levels with $d$ colors. Remove the colored vertices and consider the $2^{d} B_{k+d}$ subtrees left. To each associate an ordered set from the $[k, d, n]$ PSF family and color the whole $i^{\text {th }}$ level with one color, the $i^{\text {th }}$ element of the associated ordered set. Deleting also these colored vertices, we are left with subtrees with at most $d$ levels, which we can color with (at most) the same $d$ colors we used for the top levels. It is not difficult to check that the above procedure produces a conflict-free coloring. By repeating the above procedure $r$ times for $B_{d(r+1)+k r}$, as in corollary 3.22 , we obtain $\mathrm{CF}\left(B_{d(r+1)+k r}\right) \leq n r+d$. $\square$

Corollary 3.29. For the sequence of complete binary trees, $\left\{B_{i}\right\}_{i=1}^{\infty}$, the limit of the ratio of the UM to the CF chromatic number is at least $\log 3 \approx 1.58$.

Proof. Since $\mathrm{CF}\left(B_{d(r+1)+k r}\right) \leq n r+d$, the ratio of $\mathrm{UM}$ to $\mathrm{CF}$ for $B_{d(r+1)+k r}$ is at least $(d(r+1)+k r) /(n r+d)$, which tends to $(d+k) / n$ as $r \rightarrow \infty$. From claim 3.27 we can choose $d=\left\lfloor\log \left(\begin{array}{l}n \\ k\end{array}\right)\right\rfloor$. If we substitute $k$ with $x n$, then a short calculation shows that to maximize $(d+k) / n$ we have to maximize $x+H(x)$, where $H(x)=-x \log x-(1-x) \log (1-x)$ (entropy). The function $x+H(x)$ attains its maximum at $x=2 / 3$, giving a value of $\log 3$ as a lower bound for the limit.

For completeness, we also include a proof of the existence of the limit. For brevity, denote $\operatorname{CF}\left(B_{i}\right)$ by $c_{i}$. Our goal is to show that sequence $f$, with $f_{i}=c_{i} / i$, i.e., the ratio of $C F$ to the $U M$ for $B_{i}$ has a finite limit. If the limit exists, then it is finite, because $f_{i} \in(0,1]$. We know that $c_{i}$ is monotone increasing. We also know that $c_{i+1} \leq c_{i}+1$ since we can take two copies of a good CF-coloring of depth $i$ and join them with a root having a new color. In fact, we even know $c_{i+j} \leq c_{i}+c_{j}$ because 
we can take a good CF-coloring of depth $j$ and put $2^{j}$ copies of a good CF-coloring of depth $i$ under each of its leaves. Then, for $n \geq i$,

$$
\frac{c_{n}}{n} \leq \frac{\lceil n / i\rceil c_{i}}{n}<\left(\frac{n}{i}+1\right) \frac{c_{i}}{n}=\frac{c_{i}}{i}+\frac{c_{i}}{n} \leq \frac{c_{i}}{i}+\frac{i}{n},
$$

that is, $f_{n} \leq f_{i}+i / n$. With this, it is not difficult to prove, using standard arguments, that no two subsequences of $f$ have different limits, and thus $f$ has a limit.

REMARK 3.30. Since $\operatorname{ODD}(T) \leq \mathrm{CF}(T)$, corollary 3.29 is also true for the ratio of the UM to the ODD chromatic number. In [10], the authors prove that for the sequence of binomial trees (see remark 3.7), the ratio of the CF to the ODD chromatic number tends at least to 1.5, disproving a conjecture of [4]. Therefore, our result disproves the aforementioned conjecture in a stronger sense (our limit is at least $\log 3 \approx 1.58$ ).

4. Discussion and open problems. In the literature of conflict-free coloring, hypergraphs that are induced by geometric shapes have been in the focus. It would be interesting to show possible relations between unique-maximum and conflict-free chromatic numbers in this setting.

The exact relationship between the two chromatic numbers with respect to paths for general graphs still remains an open problem. In [6], only graphs which have unique-maximum chromatic number about twice the conflict-free chromatic number were exhibited, but the only bound proved on $\chi_{\mathrm{um}}^{\mathrm{p}}(G)$ was exponential in $\chi_{\mathrm{cf}}^{\mathrm{p}}(G)$. In fact it is even possible that $\chi_{\mathrm{um}}^{\mathrm{p}}(G) \leq 2 \chi_{\mathrm{cf}}^{\mathrm{p}}(G)-2$. The first step to prove this would be to show that $\chi_{\mathrm{um}}^{\mathrm{p}}(T)=O\left(\chi_{\mathrm{cf}}^{\mathrm{p}}(T)\right)$ for trees. It would also be interesting to extend our results to other classes of graphs.

Acknowledgment. We would like to thank Géza Tóth for fruitful discussions and ideas about improving the lower bound in corollary 3.29. We would also like to thank our reviewer for useful suggestions.

\section{REFERENCES}

[1] Amotz Bar-Noy, Panagiotis Cheilaris, and Shakhar Smorodinsky, Deterministic conflictfree coloring for intervals: from offline to online, ACM Transactions on Algorithms, 4 (2008), pp. 44.1-44.18.

[2] Hans L. Bodlaender, Jitender S. Deogun, Klaus Jansen, Ton Kloks, Dieter Kratsch, Haiko MÜller, ANd Zsolt TuzA, Rankings of graphs, SIAM Journal on Discrete Mathematics, 11 (1998), pp. 168-181.

[3] Hans L. Bodlaender, John R. Gilbert, Huálmtyr Hafsteinsson, and Ton Kloks, Approximating treewidth, pathwidth, frontsize, and shortest elimination tree, Journal of Algorithms, 18 (1995), pp. 238-255.

[4] Piotr Borowiecki, Kristína Budajová, Stanislav Jendrol', and Stanislav Krajči, Parity vertex colouring of graphs, Discussiones Mathematicae Graph Theory, 31 (2011), pp. 183195.

[5] Panagiotis Cheilaris, Conflict-free coloring, PhD thesis, City University of New York, 2009.

[6] Panagiotis Cheilaris and Géza Tóth, Graph unique-maximum and conflict-free colorings, Journal of Discrete Algorithms, 9 (2011), pp. 241-251.

[7] Ke Chen, Amos Fiat, Haim Kaplan, Meital Levy, Jiří Matoušek, Elchanan Mossel, János Pach, Micha Sharir, Shakhar Smorodinsky, Uli Wagner, and Emo Welzl, Online conflict-free coloring for intervals, SIAM Journal on Computing, 36 (2007), pp. 13421359.

[8] Khaled Elbassioni and Nabil H. Mustafa, Conflict-free colorings of rectangles ranges, in Proceedings of the 23rd International Symposium on Theoretical Aspects of Computer Science (STACS), 2006, pp. 254-263. 
[9] Guy Even, Zvi Lotker, Dana Ron, and Shakhar Smorodinsky, Conflict-free colorings of simple geometric regions with applications to frequency assignment in cellular networks, SIAM Journal on Computing, 33 (2003), pp. 94-136.

[10] Petr Gregor and Riste Šrekovski, Parity vertex colorings of binomial trees, Discussiones Mathematicae Graph Theory, 32 (2012), pp. 177-180.

[11] Sariel Har-Peled and Shakhar Smorodinsky, Conflict-free coloring of points and simple regions in the plane, Discrete and Computational Geometry, 34 (2005), pp. 47-70.

[12] Ananth V. Iyer, H. Ronald Ratliff, and Gopalakrishanan Vijayan, Optimal node ranking of trees, Information Processing Letters, 28 (1988), pp. 225-229.

[13] Meir Katchalski, William McCuaig, and Suzanne Seager, Ordered colourings, Discrete Mathematics, 142 (1995), pp. 141-154.

[14] Charles E. Leiserson, Area-efficient graph layouts (for VLSI), in Proceedings of the 21st Annual IEEE Symposium on Foundations of Computer Science (FOCS), 1980, pp. 270281.

[15] Joseph W.H. LiU, The role of elimination trees in sparse factorization, SIAM Journal on Matrix Analysis and Applications, 11 (1990), pp. 134-172.

[16] Donna Crystal Llewellyn, Craig A. Tovey, and Michael A. Trick, Local optimization on graphs., Discrete Applied Mathematics, 23 (1989), pp. 157-178.

[17] Jaroslav Nešetřil and Patrice Ossona de Mendez, Tree-depth, subgraph coloring and homomorphism bounds, European Journal of Combinatorics, 27 (2006), pp. 1022-1041.

[18] JÁnos PaCh And Gábor TARdos, Conflict-free colourings of graphs and hypergraphs, Combinatorics, Probability and Computing, 18 (2009), pp. 819-834.

[19] JÁnos PaCh And GÉza Tóth, Conflict free colorings, in Discrete and Computational Geometry, The Goodman-Pollack Festschrift, Springer Verlag, 2003, pp. 665-671.

[20] Alex Pothen, The complexity of optimal elimination trees, Tech. Report CS-88-16, Department of Computer Science, Pennsylvania State University, 1988.

[21] Shakhar Smorodinsky, Combinatorial Problems in Computational Geometry, PhD thesis, School of Computer Science, Tel-Aviv University, 2003.

[22] - Conflict-free coloring and its applications, ArXiv, abs/1005.3616 (2010). 